\title{
Closed Form expressions for moments of the beta Weibull distribution
}

\author{
GAUSS M. CORDEIRO ${ }^{1}$, ALEXANDRE B. SIMAS ${ }^{2}$ and BORKO D. STOŠIĆ \\ ${ }^{1}$ Departamento de Estatística, Universidade Federal de Pernambuco \\ Cidade Universitária, 50740-540 Recife, PE, Brasil \\ ${ }^{2}$ Departamento de Matemática, Universidade Federal da Paraíba, Cidade Universitária \\ Campus I, 58051-970 João Pessoa, PB, Brasil \\ ${ }^{3}$ Departamento de Estatística e Informática, Universidade Federal Rural de Pernambuco \\ Rua Dom Manoel de Medeiros s/n, Dois Irmãos, 52171-900 Recife, PE, Brasil \\ Manuscript received on September 15, 2009; accepted for publication on July 5, 2010
}

\begin{abstract}
The beta Weibull distribution was first introduced by Famoye et al. (2005) and studied by these authors and Lee et al. (2007). However, they do not give explicit expressions for the moments. In this article, we derive explicit closed form expressions for the moments of this distribution, which generalize results available in the literature for some sub-models. We also obtain expansions for the cumulative distribution function and Rényi entropy. Further, we discuss maximum likelihood estimation and provide formulae for the elements of the expected information matrix. We also demonstrate the usefulness of this distribution on a real data set.
\end{abstract}

Key words: beta Weibull distribution, expected information matrix, maximum likelihood, moment, Weibull distribution.

\section{INTRODUCTION}

The Weibull distribution is a popular distribution widely used for analyzing lifetime data. We work with the beta Weibull (BW) distribution because of the wide applicability of the Weibull distribution and the fact that it extends some recent developed distributions. We derive expansions for this distribution function and explicit closed form expressions for its moments. An application is illustrated to a real data set with the hope that it will attract more applications in reliability, biology and other areas of research.

The BW distribution stems from the following general class: if $G$ denotes the cumulative distribution function (cdf) of a random variable, then a generalized class of distributions can be defined by

$$
F(x)=I_{G(x)}(a, b)
$$

Correspondence to: Gauss Moutinho Cordeiro

E-mail: gausscordeiro@uol.com.br 
for $a>0$ and $b>0$, where

$$
I_{y}(a, b)=\frac{B_{y}(a, b)}{B(a, b)}=\frac{\int_{0}^{y} w^{a-1}(1-w)^{b-1} d w}{B(a, b)}
$$

is the incomplete beta function ratio, $B_{y}(a, b)$ is the incomplete beta function, $B(a, b)=\Gamma(a) \Gamma(b) /$ $\Gamma(a+b)$ is the beta function, and $\Gamma(\cdot)$ is the gamma function. This class of generalized distributions has been receiving increased attention over the last years, in particular after the recent works of Eugene et al. (2002) and Jones (2004). Eugene et al. (2002) introduced which is known as the beta normal distribution by taking $G(x)$ in (1) to be the cumulative function of the normal distribution with parameters $\mu$ and $\sigma$. The only properties of the beta normal distribution known are some first moments derived by Eugene et al. (2002) and some more general moment expressions derived by Gupta and Nadarajah (2004). More recently, Nadarajah and Kotz (2004) were able to provide closed form expressions for the moments, the asymptotic distribution of the extreme order statistics and the estimation procedure for the beta Gumbel distribution. Another distribution that happens to belong to (1) is the $\log (F)$ (or beta logistic) distribution, which has been around for over 20 years (Brown et al. 2002), even if it did not originate directly from (1).

While the transformation (1) is not analytically tractable in the general case, the formulae related with the BW are manageable (as it is shown in this paper), and with the use of modern computer resources with analytic and numerical capabilities, may turn into adequate tools comprising the arsenal of applied statisticians. The current work represents an advance in the direction traced by Nadarajah and Kotz (2006), contrary to their belief that some mathematical properties of the BW distribution are not tractable.

Replacing $G(x)$ by the cdf of the Weibull distribution with parameters $c$ and $\lambda$ in (1) yields the cdf of the $\mathrm{BW}$ distribution

$$
F(x)=I_{1-\exp \left\{-(\lambda x)^{c}\right\}}(a, b)
$$

for $x>0, a>0, b>0, c>0$ and $\lambda>0$. The probability density function (pdf) and the hazard rate function corresponding to (2) are

$$
f(x)=\frac{c \lambda^{c}}{B(a, b)} x^{c-1} \exp \left\{-b(\lambda x)^{c}\right\}\left[1-\exp \left\{-(\lambda x)^{c}\right\}\right]^{a-1}
$$

and

$$
\tau(x)=\frac{c \lambda^{c} x^{c-1} \exp \left\{-b(\lambda x)^{c}\right\}\left[1-\exp \left\{-(\lambda x)^{c}\right\}\right]^{a-1}}{B_{1-\exp \left\{-(\lambda x)^{c}\right\}}(a, b)},
$$

respectively. Simulation from (3) is easy: if $B$ is a random number following a beta distribution with parameters $a$ and $b$, then $X=\{-\log (1-B)\}^{1 / c} / \lambda$ will follow a BW distribution with parameters $a, b, c$ and $\lambda$. Some mathematical properties of the BW distribution are given by Famoye et al. (2005) and Lee et al. (2007).

Graphical representations of equations (3) and (4) for some choices of parameters $a$ and $b$, fixed $c=3$ and $\lambda=1$, are given in Figures 1 and 2, respectively. The Weibull distribution corresponds to the particular case $a=b=1$. Thus, the Weibull distribution is generalized by a family of curves shown in these figures with a variety of shapes.

The rest of the paper is organized as follows. In Section 2, we derive some expansions for the cdf of the BW distribution, and point out some special cases that have been considered in the literature. 


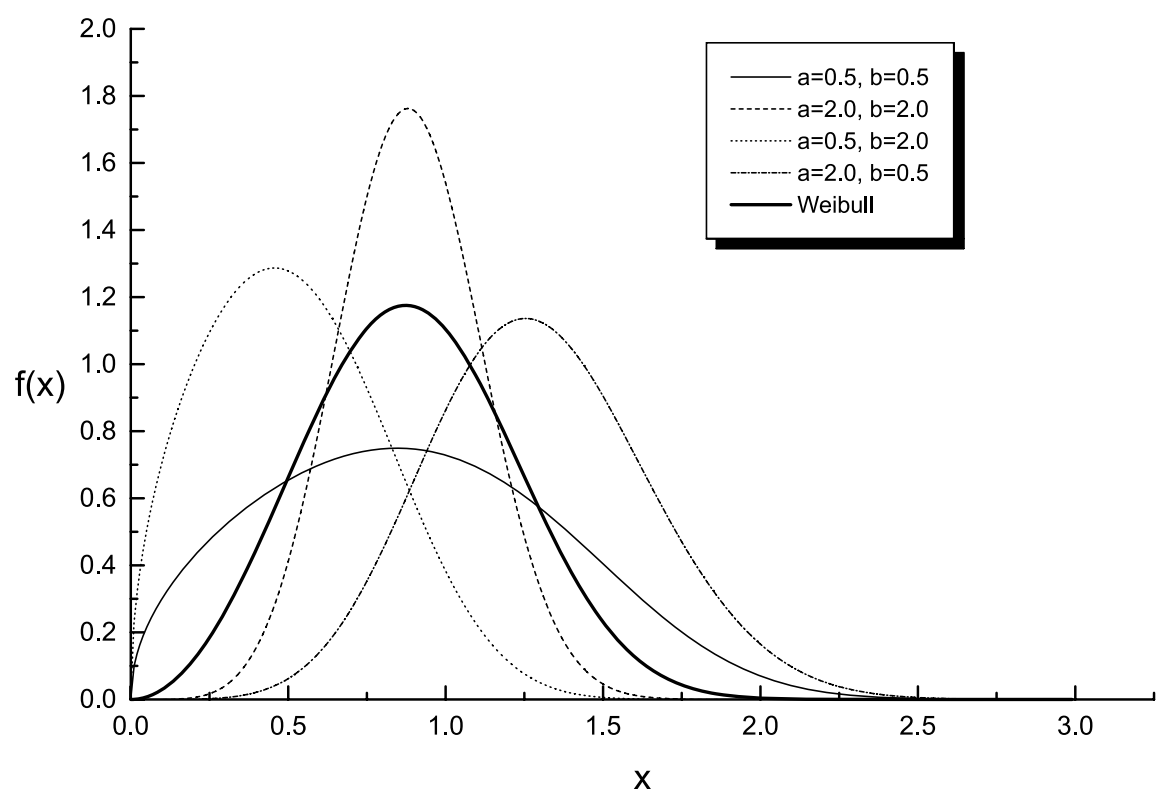

Fig. 1 - The BW density function (3) for selected values of parameters $a$ and $b$.

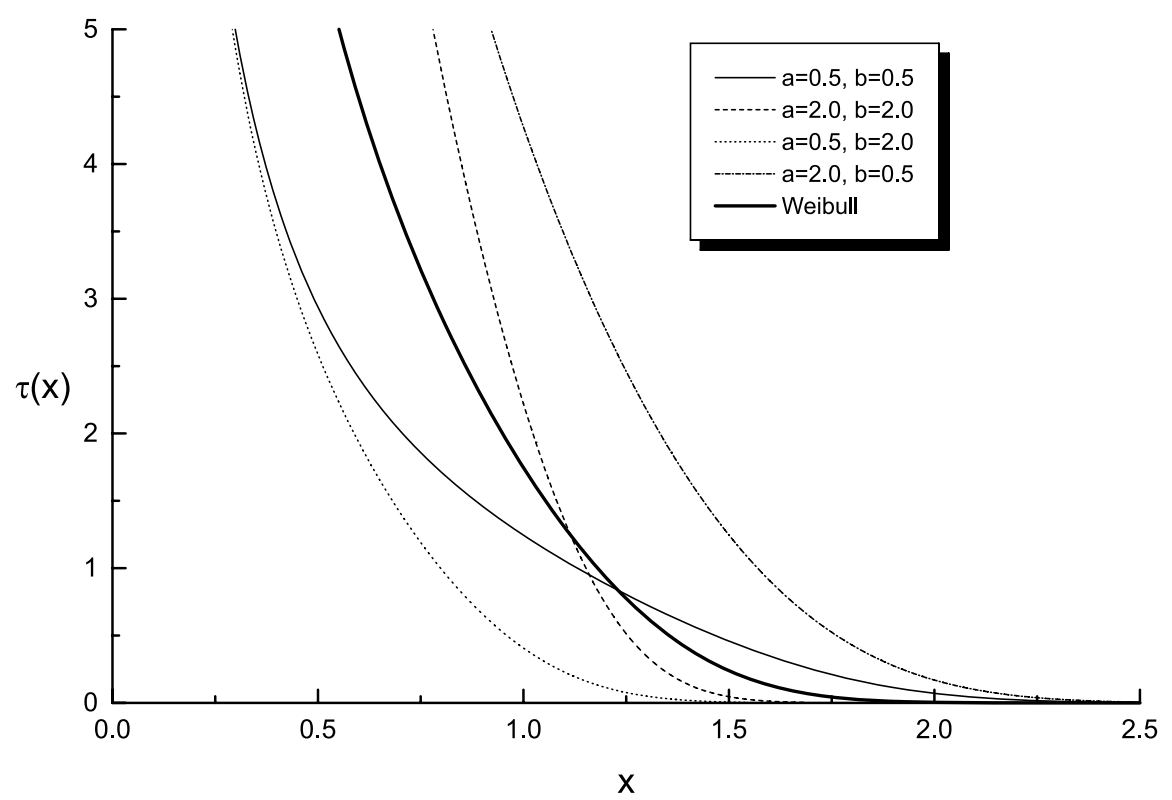

Fig. 2 - The BW hazard function (4) for selected values of parameters $a$ and $b$.

In Section 3, we obtain explicit closed form expressions for the moments. We also present plots of the skewness and kurtosis for selected parameter values. Section 4 provides an expansion for the moment generating function (mgf). In Section 5, we obtain a closed-form expression for the Rényi entropy. In Section 6, we discuss the maximum likelihood estimation and provide the elements of the Fisher information matrix. In Section 7, an application to real data is presented. Section 8 deals with a log-beta Weibull distribution. In Section 9, we provide some conclusions. In the Appendix, two identities required in Section 3 are derived. 


\section{EXPANSIONS FOR THE DISTRIBUTION FUNCTION}

The BW distribution is an extended model to analyze more complex data and generalizes some recent distributions in the literature. In particular, the BW distribution contains the exponentiated Weibull distribution (for instance, see Mudholkar et al. 1995, Mudholkar and Hutson 1996, Nassar and Eissa 2003, Nadarajah and Gupta 2005, Choudhury 2005) as special cases when $b=1$. The Weibull distribution (with parameters $c$ and $\lambda$ ) is clearly a special case for $a=b=1$. When $a=1$, (3) follows a Weibull distribution with parameters $\lambda b^{1 / c}$ and $c$. The beta exponential distribution (Nadarajah and Kotz 2006) is also a special case for $c=1$.

In what follows, we provide two simple formulae for the $\operatorname{cdf}(2)$, depending on whether the parameter $a>0$ is a real non-integer or an integer, which may be used for further analytical or numerical analysis. Starting from the explicit expression for the cdf (2)

$$
F(x)=\frac{c \lambda^{c}}{B(a, b)} \int_{0}^{x} y^{c-1} \exp \left\{-b(\lambda y)^{c}\right\}\left[1-\exp \left\{-(\lambda y)^{c}\right\}\right]^{a-1} d y
$$

and setting $(\lambda y)^{c}=u$ gives

$$
F(x)=\frac{1}{B(a, b)} \int_{0}^{(\lambda x)^{c}} e^{-b u}\left(1-e^{-u}\right)^{a-1} d u .
$$

If $a>0$ is a real non-integer, we have

$$
(1-z)^{a-1}=\sum_{j=0}^{\infty} \frac{(-1)^{j} \Gamma(a) z^{j}}{\Gamma(a-j) j !} .
$$

It follows that

$$
\begin{aligned}
F(x) & =\frac{1}{B(a, b)} \int_{0}^{(\lambda x)^{c}} e^{-b u} \sum_{j=0}^{\infty} \frac{(-1)^{j} \Gamma(a) e^{-j u}}{\Gamma(a-j) j !} d u \\
& =\frac{1}{B(a, b)} \sum_{j=0}^{\infty} \frac{(-1)^{j} \Gamma(a)}{\Gamma(a-j) j !} \int_{0}^{(\lambda x)^{c}} e^{-(b+j) u} d u \\
& =\frac{1}{B(a, b)} \sum_{j=0}^{\infty} \frac{(-1)^{j} \Gamma(a)}{\Gamma(a-j) j !(b+j)}\left\{1-e^{-(b+j)(\lambda x)^{c}}\right\}
\end{aligned}
$$

Hence, we obtain

$$
F(x)=\frac{\Gamma(a+b)}{\Gamma(b)} \sum_{j=0}^{\infty} \frac{(-1)^{j}\left\{1-e^{-(b+j)(\lambda x)^{c}}\right\}}{\Gamma(a-j) j !(b+j)} .
$$

For positive real non-integer $a$, the expansion (6) may be used for further analytical and/or numerical studies. For integer $a$, we change the formula in (5) to the binomial expansion to obtain

$$
F(x)=\frac{1}{B(a, b)} \sum_{j=0}^{a-1}\left(\begin{array}{c}
a-1 \\
j
\end{array}\right) \frac{(-1)^{j}\left\{1-e^{-(b+j)(\lambda x)^{c}}\right\}}{(b+j)} .
$$


When both $a$ and $b=n-a+1$ are integers, the relation of the incomplete beta function to the binomial expansion yields

$$
F(x)=\sum_{j=a}^{n}\left(\begin{array}{l}
n \\
j
\end{array}\right)\left[1-\exp \left\{-(\lambda x)^{c}\right\}\right]^{j} \exp \left\{-(n-j)(\lambda x)^{c}\right\} .
$$

It can be found in the Wolfram Functions Site $^{1}$ that, for integer $a$,

$$
I_{y}(a, b)=1-\frac{(1-y)^{b}}{\Gamma(b)} \sum_{j=0}^{a-1} \frac{\Gamma(b+j) y^{j}}{j !}
$$

and for integer $b$,

$$
I_{y}(a, b)=\frac{y^{a}}{\Gamma(a)} \sum_{j=0}^{b-1} \frac{\Gamma(a+j)(1-y)^{j}}{j !} .
$$

Hence, if $a$ is an integer, we have another equivalent form for (7)

$$
F(x)=1-\frac{\exp \left\{-b(\lambda x)^{c}\right\}}{\Gamma(b)} \sum_{j=0}^{a-1} \frac{\Gamma(b+j)}{j !}\left[1-\exp \left\{-(\lambda x)^{c}\right\}\right]^{j} .
$$

For integer values of $b$, we obtain

$$
F(x)=\frac{\left[1-\exp \left\{-(\lambda x)^{c}\right\}\right]^{a}}{\Gamma(a)} \sum_{j=0}^{b-1} \frac{\Gamma(a+j)}{j !} \exp \left\{-j(\lambda x)^{c}\right\} .
$$

Finally, if $a=1 / 2$ and $b=1 / 2$, we have

$$
F(x)=\frac{2}{\pi} \arctan \sqrt{\exp \left\{(\lambda x)^{c}\right\}-1} .
$$

The special cases (8) and (9) were discussed generally by Jones (2004), and the expansions (6) and (10) reduce to Nadarajah and Kotz's (2006) results for the beta exponential distribution by setting $c=1$. Clearly, the expansions for the BW density function are obtained from (6) and (7) by simple differentiation. Hence, the BW density function can be expressed in a mixture form of Weibull density functions.

\section{MOMENTS}

The moments are used for computing skewness and kurtosis and for computing moment estimators (MM), which could at least be used as starting values for maximum likelihood estimation. Let $X$ be a BW random variable following the density function (3). We derive explicit expressions for the moments of $X$. First, we introduce the following notation (for any real $d$ and $a$ and $b$ positive)

$$
S_{d, b, a}=\int_{0}^{\infty} x^{d-1} \exp (-b x)\{1-\exp (-x)\}^{a-1} d x
$$

\footnotetext{
1 http://functions.wolfram.com/GammaBetaErf/BetaRegularized/03/01/
} 
The change of variables $x=-\log (z)$ immediately yields $S_{1, b, a}=B(a, b)$. On the other hand, the change of variables $x=(\lambda y)^{c}$ gives the following relation

$$
\int_{0}^{\infty} y^{\gamma-1} \exp \left\{-b(\lambda y)^{c}\right\}\left[1-\exp \left\{-(\lambda y)^{c}\right\}\right]^{a-1} d y=\frac{\lambda^{-\gamma}}{c} S_{\frac{\gamma}{c}, b, a},
$$

from which it follows that

$$
S_{\gamma / c, b, a}=B(a, b) \lambda^{\gamma-c} E\left(X^{\gamma-c}\right),
$$

or, equivalently, for any real $r$,

$$
E\left(X^{r}\right)=\frac{1}{\lambda^{r} B(a, b)} S_{r / c+1, b, a},
$$

relating $S_{r / c+1, b, a}$ to the $r$ th generalized moment of the BW distribution.

First, we consider the integral (11) when $d$ is an integer. Let $U$ be a random variable following the $\operatorname{Beta}(b, a)$ distribution with pdf $f_{U}(\cdot)$ and $W=-\log (U)$. Further, let $F_{U}(\cdot)$ and $F_{W}(\cdot)$ be the cdfs of $U$ and $W$, respectively. It is easy to see that $F_{W}(x)=1-F_{U}\left(e^{-x}\right)$. Further, some properties of the Lebesgue-Stiltjes integral lead to

$$
\begin{aligned}
E\left(W^{d-1}\right) & =\int_{-\infty}^{\infty} x^{d-1} d F_{W}(x)=\int_{0}^{\infty} x^{d-1} e^{-x} f_{U}\left(e^{-x}\right) d x \\
& =\frac{1}{B(a, b)} \int_{0}^{\infty} x^{d-1} e^{-b x}\left(1-e^{-x}\right)^{a-1} d x=\frac{S_{d, b, a}}{B(a, b)} .
\end{aligned}
$$

Thus, the values of $S_{d, b, a}$ for integer values of $d$ can be found from the moments of $W$ if they are known. However, the moment generating function (mgf) $M_{W}(t)=E\left(e^{t W}\right)$ of $W$ can be expressed as

$$
M_{W}(t)=E\left(U^{-t}\right)=\frac{1}{B(a, b)} \int_{0}^{1} x^{b-t-1}(1-x)^{a-1} d x=\frac{B(b-t, a)}{B(a, b)} .
$$

This formula is well defined for $t<b$. However, we are only interested in the limit $t \rightarrow 0$ and, therefore, this expression can be used for the current purpose. We can write

$$
S_{d, b, a}=B(a, b) E\left(W^{d-1}\right)=B(a, b) M_{W}^{(d-1)}(0)=\left.\frac{\partial^{d-1}}{\partial t^{d-1}} B(b-t, a)\right|_{t=0} .
$$

From equations (13) and (14), for any positive integer $k$, we obtain a general formula

$$
E\left(X^{k c}\right)=\left.\frac{1}{\lambda^{k c} B(a, b)} \frac{\partial^{k}}{\partial t^{k}} B(b-t, a)\right|_{t=0} .
$$

As particular cases, we can see directly from equation (14) that

$$
S_{1, b, a}=B(a, b), S_{2, b, a}=B(a, b)\{\psi(a+b)-\psi(b)\}
$$

and

$$
S_{3, b, a}=B(a, b)\left[\psi^{\prime}(b)-\psi^{\prime}(a+b)+\{\psi(a+b)-\psi(b)\}^{2}\right],
$$

and using (13), we obtain

$$
E\left(X^{c}\right)=\frac{\{\psi(a+b)-\psi(b)\}}{\lambda^{c}}
$$


and

$$
E\left(X^{2 c}\right)=\frac{\psi^{\prime}(b)-\psi^{\prime}(a+b)+\{\psi(a+b)-\psi(b)\}^{2}}{\lambda^{2 c}} .
$$

The same results can also be obtained directly from equation (15).

Note that the formula for $S_{1, b, a}$ matches the one just given after equation (12). Since the BW distribution for $c=1$ reduces to the beta exponential distribution, the above formulae for $E\left(X^{c}\right)$ and $E\left(X^{2 c}\right)$ reduce to the corresponding ones obtained by Nadarajah and Kotz (2006).

Our main goal here is to give the $r$ th moment of $X$ for every positive integer $r$. In fact, in what follows, we obtain the $r$ th generalized moment for every real $r$, which may be used for further theoretical or numerical analysis. To this end, we need to obtain a formula for $S_{d, b, a}$ that holds for every positive real $d$. In the appendix we show that, for any $d>0$, the following identity holds for positive real non-integer $a$

$$
S_{d, b, a}=\Gamma(a) \Gamma(d) \sum_{j=0}^{\infty} \frac{(-1)^{j}}{\Gamma(a-j) j !(b+j)^{d}}
$$

and that, when $a$ is positive integer,

$$
S_{d, b, a}=\Gamma(d) \sum_{j=0}^{a-1}\left(\begin{array}{c}
a-1 \\
j
\end{array}\right) \frac{(-1)^{j}}{(b+j)^{d}}
$$

is satisfied.

From equations (13) and (16), the $r$ th generalized moment of $X$ for positive real non-integer $a$ can be written as

$$
E\left(X^{r}\right)=\frac{\Gamma(a) \Gamma(r / c+1)}{\lambda^{r} B(a, b)} \sum_{j=0}^{\infty} \frac{(-1)^{j}}{\Gamma(a-j) j !(b+j)^{r / c+1}} .
$$

When $a>0$ is an integer, we obtain

$$
E\left(X^{r}\right)=\frac{\Gamma(r / c+1)}{\lambda^{r} B(a, b)} \sum_{j=0}^{a-1}\left(\begin{array}{c}
a-1 \\
j
\end{array}\right) \frac{(-1)^{j}}{(b+j)^{r / c+1}} .
$$

When $a=b=1, X$ follows a Weibull distribution and formula (19) reduces to

$$
E\left(X^{r}\right)=\frac{\Gamma(r / c+1)}{\lambda^{r}}
$$

which is precisely the $r$ th moment of the Weibull distribution with parameters $\lambda$ and $c$. Equations (15), (18) and (19) represent the main results of this section, which may serve as a starting point for applications in particular cases, as well as for further research.

Graphical representations of skewness and kurtosis for some choices of parameter $b$ as a function of parameter $a$, and for some choices of parameter $a$ as a function of parameter $b$, for fixed $\lambda=1$ and $c=3$, are given in Figures 3 and 4, and 5 and 6, respectively. Figures 3 and 4 show that the skewness and kurtosis curves cross at $a=1$, whereas Figures 5 and 6 show that both skewness and kurtosis are independent of $b$ for $a=1$. In addition, the Weibull distribution $(a=b=1)$ appears as a single point in Figures 3-6. 


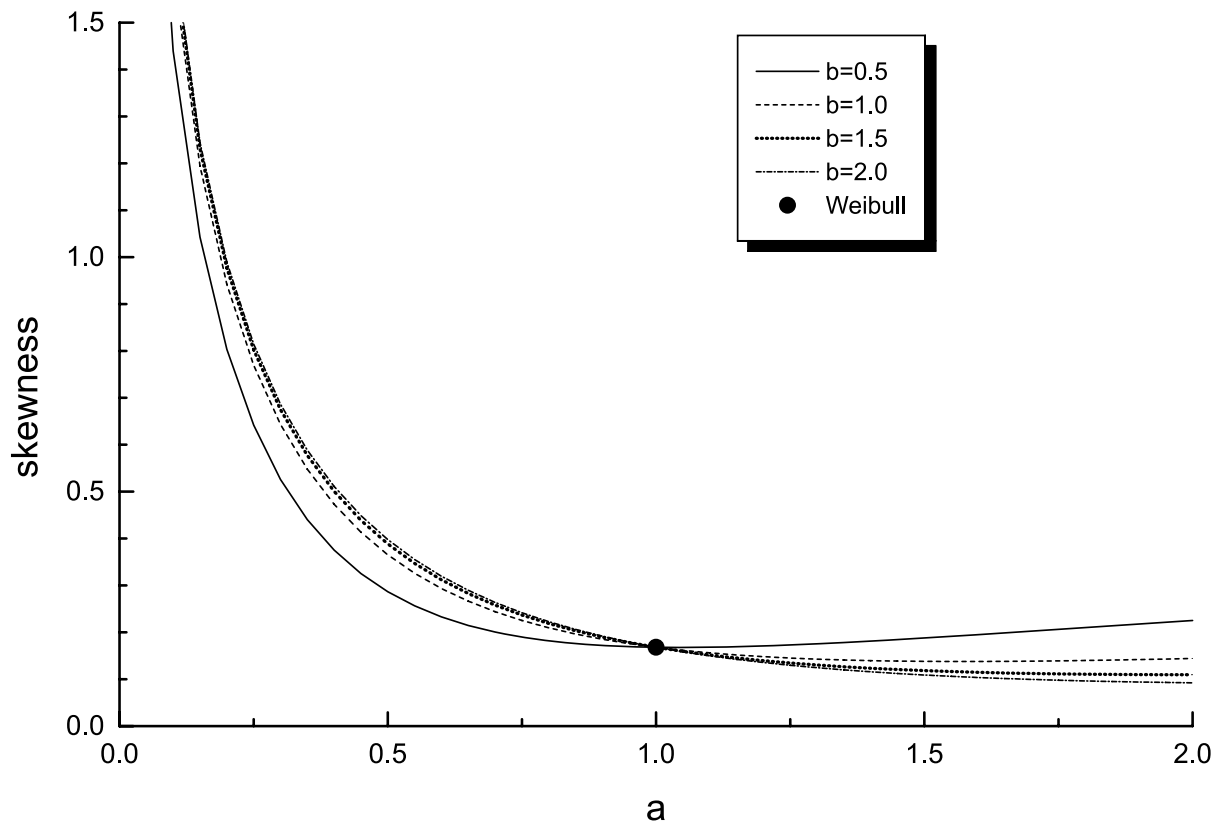

Fig. 3 - Skewness of the $\mathrm{BW}$ distribution as a function of parameter $a$ for selected values of parameter $b$.

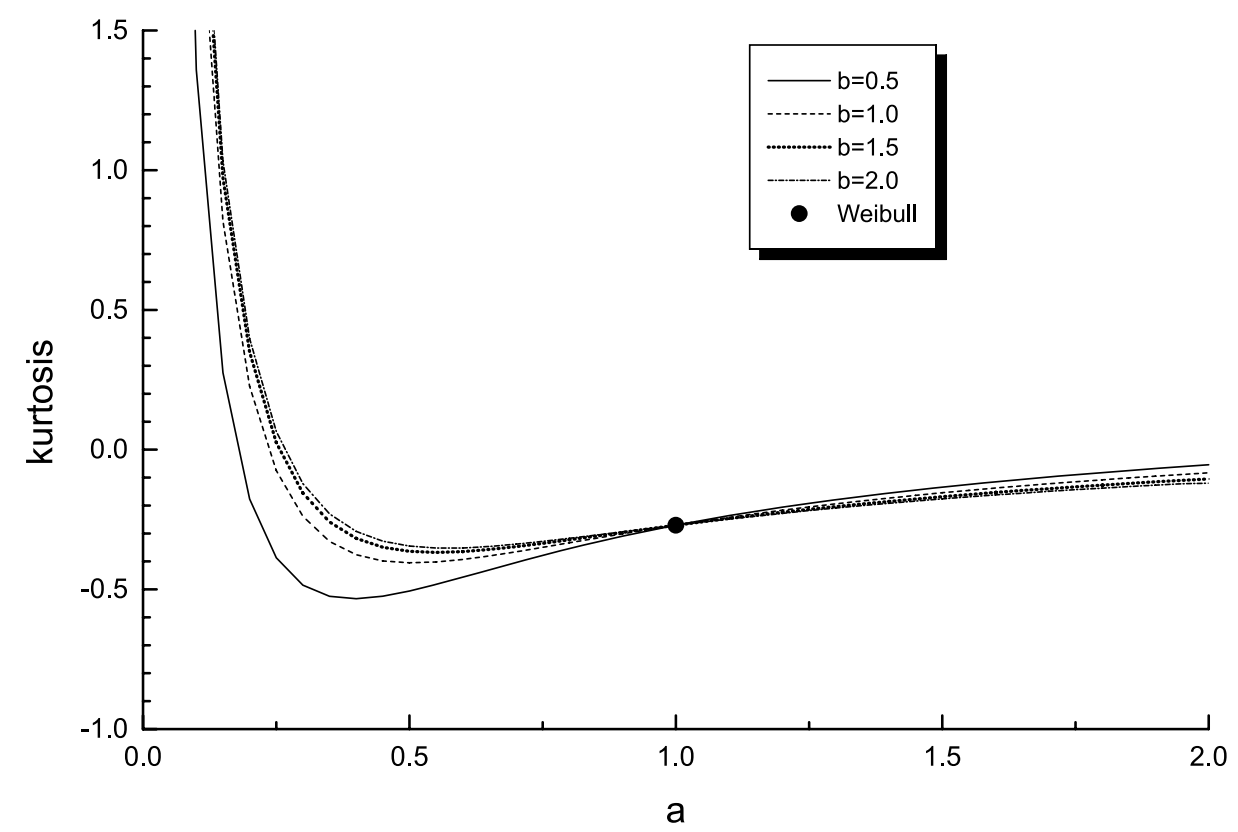

Fig. 4 - Kurtosis of the BW distribution as a function of parameter $a$ for selected values of parameter $b$.

We now consider the estimation of the model parameters by the methods of moments. For the moments estimation, let

$$
m_{k}=(1 / n) \sum_{j=1}^{n} t_{j}^{k} \quad \text { for } \quad k=1, \ldots, 4
$$




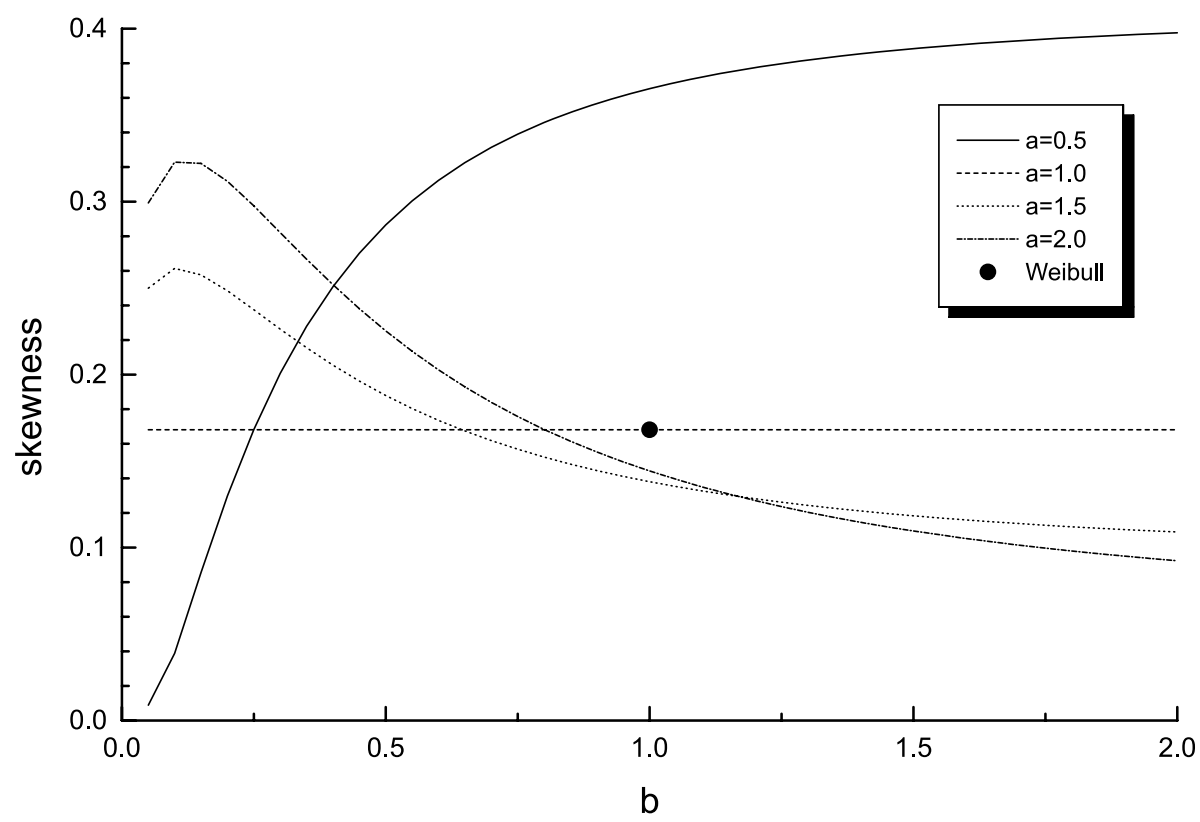

Fig. 5 - Skewness of the BW distribution as a function of parameter $b$ for selected values of parameter $a$.

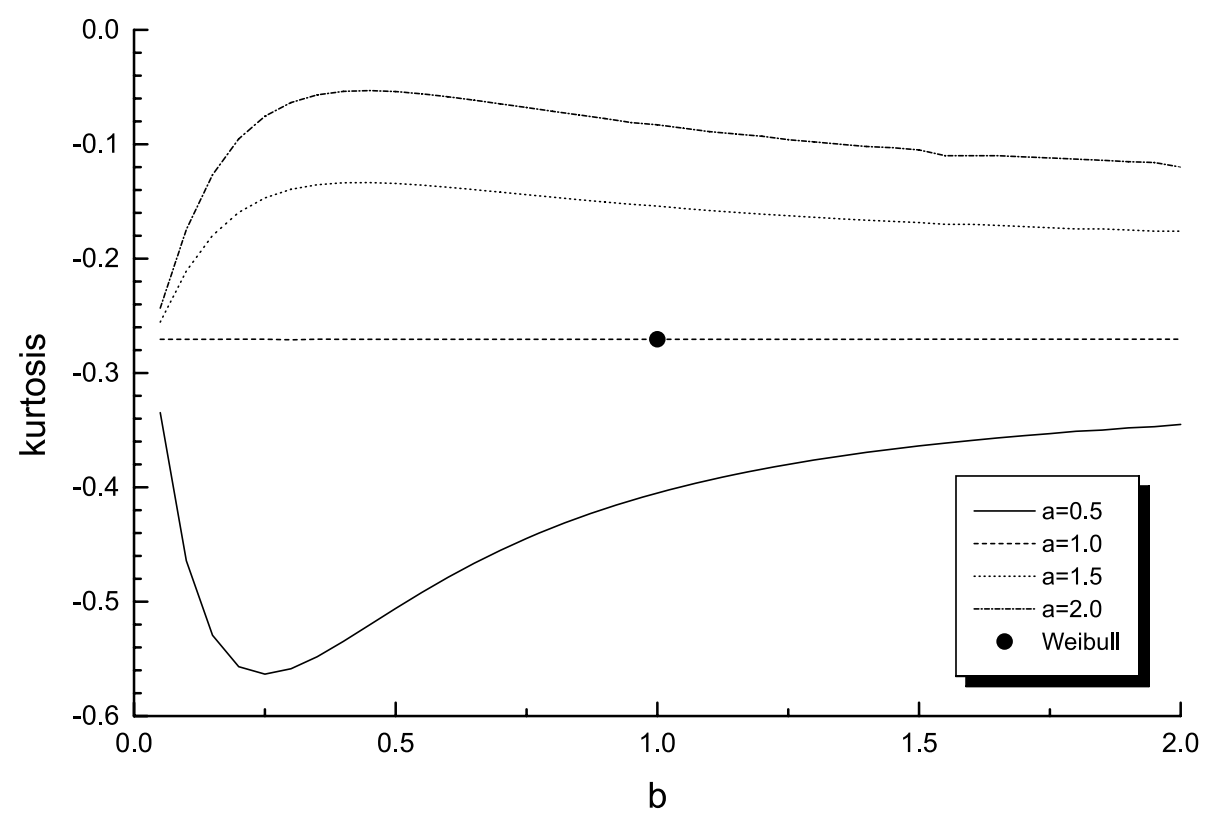

Fig. 6 - Kurtosis of the BW distribution as a function of parameter $b$ for selected values of parameter $a$.

By equating the theoretical moments (18) with the sample moments, we obtain the equations:

$$
\frac{\Gamma(a) \Gamma(r / c+1)}{\lambda^{r} B(a, b)} \sum_{j=0}^{\infty} \frac{(-1)^{j}}{\Gamma(a-j) j !(b+j)^{r / c+1}}=m_{r}
$$

for $r=1, \ldots, 4$. The method of moments estimators is the simultaneous solutions of these equations. 


\section{MOMENT GENERATING FUNCTION}

Here, we provide an expansion for the mgf of the BW distribution. We have

$$
\begin{aligned}
M(t) & =\frac{c \lambda^{c}}{B(a, b)} \int_{0}^{\infty} \exp (t x) x^{c-1} \exp \left\{-b(\lambda x)^{c}\right\}\left[1-\exp \left\{-(\lambda x)^{c}\right\}\right]^{a-1} d x \\
& =\frac{c \lambda^{c}}{B(a, b)} \sum_{r=0}^{\infty} \frac{t^{r}}{r !} \int_{0}^{\infty} x^{r+c-1} \exp \left\{-b(\lambda x)^{c}\right\}\left[1-\exp \left\{-(\lambda x)^{c}\right\}\right]^{a-1} d x \\
& =\frac{c \lambda^{c}}{B(a, b)} \sum_{r=0}^{\infty} \frac{t^{r}}{r !} \frac{\lambda^{-(r+c)}}{c} S_{r / c+1, b, a},
\end{aligned}
$$

where the last expression comes from equation (12). For real non-integer $a>0$, we obtain from equation (16)

$$
M(t)=\frac{\Gamma(a)}{B(a, b)} \sum_{r=0}^{\infty} \sum_{j=0}^{\infty} \frac{t^{r} \Gamma(r / c+1)(-1)^{j}}{\lambda^{r} \Gamma(a-j)(b+j)^{r / c+1} r ! j !},
$$

and for integer $a>0$ using (17),

$$
M(t)=\frac{1}{B(a, b)} \sum_{r=0}^{\infty} \frac{t^{r} \Gamma(r / c+1)}{\lambda^{r} r !} \sum_{j=0}^{a-1}\left(\begin{array}{c}
a-1 \\
j
\end{array}\right) \frac{(-1)^{j}}{(b+j)^{r / c+1}} .
$$

The expression for the mgf obtained by Choudhury (2005) is a particular case of equation (20) corresponding to $a=\theta, \lambda=1 / \alpha$ and $b=1$. When $c=1$, we have

$$
M(t)=\sum_{r=0}^{\infty} \frac{\lambda^{-r} t^{r}}{c B(a, b) r !} S_{r+1, b, a}=\frac{\lambda}{B(a, b)} \int_{0}^{\infty} e^{t x-b \lambda x}\left(1-e^{-\lambda x}\right)^{a-1} d x .
$$

Setting $y=\exp (-\lambda x)$ in the above integral yields

$$
M(t)=\frac{1}{B(a, b)} \int_{0}^{1} y^{b-t / \lambda-1}(1-y)^{a-1} d y,
$$

and using the definition of the beta function in equation (21) yields

$$
M(t)=\frac{B(b-t / \lambda, a)}{B(a, b)},
$$

which is precisely the expression (3.1) obtained by Nadarajah and Kotz (2006).

\section{RÉNYI ENTROPY}

The entropy of a random variable $X$ is a measure of uncertainty variation. The Rényi entropy is defined as $I_{R}(\delta)=(1-\delta)^{-1} \log \left\{\int_{\mathbb{R}} f^{\delta}(x) d x\right\}$, where $\delta>0$ and $\delta \neq 1$. We have

$$
f^{\gamma}(x)=\frac{c^{\gamma} \lambda^{c \gamma}}{B^{\gamma}(a, b)} x^{\gamma(c-1)} e^{-b \gamma(\lambda x)^{c}}\left\{1-e^{-(\lambda x)^{c}}\right\}^{\gamma(a-1)}
$$


and, if $\gamma(a-1)+1>0$ is a real non-integer, we expand $\left\{1-e^{-(\lambda x)^{c}}\right\}^{\gamma(a-1)}$ as in (5) to obtain

$$
f^{\gamma}(x)=\frac{c^{\gamma} \lambda^{c \gamma}}{B^{\gamma}(a, b)} \sum_{j=0}^{\infty} \frac{(-1)^{j} \Gamma(\gamma(a-1)+1)}{\Gamma(\gamma(a-1)+1-j) j !} x^{\gamma(c-1)} e^{-(b \gamma+j)(\lambda x)^{c}} .
$$

If $\gamma(a-1)+1>0$ is an integer, the sum stops at $\gamma(a-1)$. Thus, if $(\gamma-1)(c-1)>0$, an expression for Rényi entropy is given by

$$
\begin{aligned}
I_{R}(\delta)= & \frac{1}{1-\delta}\{(\gamma-1) \log (c \lambda)+\gamma \log B(a, b)+\log \Gamma((\gamma-1)(c-1) / c+1) \\
& \left.+\log \Gamma(\gamma(a-1)+1)+\log \left[\sum_{j=0}^{\infty} \frac{(-1)^{j}(b \gamma+j)^{(\gamma-1)(c-1) / c+1}}{\Gamma(\gamma(a-1)+1-j) j !}\right]\right\} .
\end{aligned}
$$

It is important to derive an expression for Rényi entropy because it serves as a measure of shape of a distribution and can be used to compare the tails and shapes of various frequently used densities, whereas the traditional kurtosis measure is not applicable; for further details, see Song (2001).

\section{ESTIMATION AND INFORMATION MATRIX}

Let $Y$ be a random variable with the BW distribution (3). The log-likelihood for a single observation $y$ of $Y$ is given by

$$
\begin{aligned}
\ell(\lambda, c, a, b)= & \log (c)+c \log (\lambda)+(c-1) \log (y)-\log \{B(a, b)\}-b(\lambda y)^{c} \\
& +(a-1) \log \left[1-\exp \left\{-(\lambda y)^{c}\right\}\right] .
\end{aligned}
$$

The corresponding components of the score vector are:

$$
\begin{gathered}
\frac{\partial \ell}{\partial a}=-\{\psi(a)-\psi(a+b)\}+\log \left\{1-e^{-(\lambda y)^{c}}\right\}, \\
\frac{\partial \ell}{\partial b}=-\{\psi(b)-\psi(a+b)\}-(\lambda y)^{c}, \\
\frac{\partial \ell}{\partial c}=\frac{1}{c}+\log (\lambda y)-b(\lambda y)^{c} \log (\lambda y)+\frac{(a-1)(\lambda y)^{c} \log (\lambda y) e^{-(\lambda y)^{c}}}{1-e^{-(\lambda y)^{c}}}
\end{gathered}
$$

and

$$
\frac{\partial \ell}{\partial \lambda}=\frac{c}{\lambda}-\frac{b c}{\lambda}(\lambda y)^{c}+\frac{c(a-1)(\lambda y)^{c} e^{-(\lambda y)^{c}}}{\lambda\left\{1-e^{-(\lambda y)^{c}}\right\}} .
$$

The maximum likelihood estimates (MLEs) can be calculated by making equations (22)-(25) equal to zero. These equations can be solved numerically for $a, b, c$ and $\lambda$. We can use iterative techniques such as a Newton-Raphson type algorithm to obtain the estimates of these parameters. From $E(\partial \ell / \partial b)=0$, equation (23) yields

$$
E\left(Y^{c}\right)=\frac{\psi(a+b)-\psi(b)}{\lambda^{c}},
$$

which agrees with the previous calculations. 
For interval estimation of $(a, b, c, \lambda)$ and hypothesis tests, the expected information matrix is required. For expressing the elements of this matrix, it is convenient to introduce an extension of the integral (11)

$$
T_{d, b, a, e}=\int_{0}^{\infty} x^{d-1} e^{-b x}\left(1-e^{-x}\right)^{a-1}(\log x)^{e} d x
$$

so that we have

$$
T_{d, b, a, 0}=S_{d, b, a} .
$$

As before, let $W=-\log (U)$, where $U$ is a random variable following the $\operatorname{Beta}(b, a)$ distribution. Then,

$$
\begin{aligned}
E\left[W^{d-1}\{\log (W)\}^{e}\right] & =\int_{-\infty}^{\infty} x^{d-1}(\log x)^{e} d F_{W}(x) \\
& =\frac{1}{B(a, b)} \int_{0}^{\infty} x^{d-1} e^{b x}\left(1-e^{-x}\right)^{a-1}(\log x)^{e} d x \\
& =\frac{T_{d, b, a, e}}{B(a, b)}
\end{aligned}
$$

Hence, the equation

$$
T_{d, b, a, e}=B(a, b) E\left[W^{d-1}\{\log (W)\}^{e}\right],
$$

relates the integral $T_{d, b, a, e}$ to expected values.

To simplify the expressions for some elements of the expected information matrix, the following identities are useful:

$$
S_{1, b+2, a}-2 S_{1, b+1, a}+S_{1, b, a}=B(a+2, b)
$$

and

$$
b S_{2, b, a}-(a+2 b+1) S_{2, b+1, a}+(a+b+1) S_{2, b+2, a}=B(a+2, b),
$$

which can be easily proved.

Explicit expressions for the elements of the information matrix $K$, obtained using Maple and Mathematica algebraic manipulation software (we have used both for double checking the obtained expressions), are given below in terms of the integrals (11) and (26):

$$
\begin{aligned}
\kappa_{a, a}= & \psi^{\prime}(a)-\psi^{\prime}(a+b), \\
\kappa_{a, b}= & -\psi^{\prime}(a+b), \kappa_{a, c}=-\frac{T_{2, b+1, a-1,1}}{c B(a, b)}, \kappa_{a, \lambda}=-\frac{c S_{2, b+1, a-1}}{\lambda B(a, b)}, \\
\kappa_{b, b}= & \psi^{\prime}(b)-\psi^{\prime}(a+b), \kappa_{b, c}=\frac{T_{2, b, a, 1}}{c B(a, b)}, \kappa_{b, \lambda}=\frac{c}{\lambda} \frac{S_{2, b, a}}{B(a, b)}, \\
\kappa_{c, c}= & \frac{1}{c^{2}}+\frac{1}{c^{2} B(a, b)}\left\{(a-1) T_{3, b+1, a-2,2}+b T_{2, b, a-2,2}\right. \\
& \left.T_{2, b+1, a-2,2}+(a+b-1) T_{2, b+2, a-2,2}\right\},
\end{aligned}
$$




$$
\begin{aligned}
\kappa_{c, \lambda}= & \frac{1}{\lambda B(a, b)}\left\{(a-1) T_{3, b+1, a-2,1}+b T_{2, b, a-2,1}\right. \\
& \left.-(a+2 b-1) T_{2, b+1, a-2,1}+(a+b-1) T_{2, b+2, a-2,1}\right\}
\end{aligned}
$$

and

$$
\kappa_{\lambda, \lambda}=\frac{c^{2}}{\lambda^{2}}+\frac{c^{2}(a-1)}{\lambda^{2} B(a, b)} S_{3, b+1, a-2} .
$$

The integrals $S_{i, j, k}$ and $T_{i, j, k, l}$ in the expected information matrix are easily determined numerically using MAPLE and MATHEMATICA for any parameter values.

The observed information matrix could also replace the expected information matrix. The latter would possibly not depend on complicated integral computations and could be used in interval and testing implementation. Second derivatives for the log-likelihood could also be numerically computed.

Under the conditions that are fulfilled for parameters in the interior of the parameter space, but not on the boundary, the asymptotic distribution of the MLEs $\hat{a}, \hat{b}, \hat{c}$ and $\hat{\lambda}$ is multivariate normal $N_{4}\left(0, K^{-1}\right)$. The estimated multivariate normal $N_{4}\left(0, \widehat{K}^{-1}\right)$ distribution can be used to construct approximate confidence intervals and confidence regions for the individual parameters and for the hazard rate and survival functions. The likelihood ratio (LR) statistic is useful for comparing the BW distribution with some of its special sub-models.

We can compute the maximum values of the unrestricted and restricted log-likelihoods to construct the LR statistics for testing some sub-models of the BW distribution. For example, we may use the LR statistic to check if the fit using the BW distribution is statistically "superior" to a fit using the exponentiated Weibull (EW) or Weibull distributions for a given data set. Mudholkar et al. (1995), in their discussion of the classical bus-motor-failure data, noted the curious aspect in which the larger EW distribution provides an inferior fit as compared to the smaller Weibull distribution.

\section{APPLICATION TO REAL DATA}

In this section we compare the results of fitting the BW and Weibull distributions to the data set studied by Meeker and Escobar (1998, p. 383), who give the times of failure and running for a sample of devices from a field-tracking study of a larger system. At a certain point in time, 30 units were installed in normal service conditions. Two causes of failure were observed for each unit that failed: the failure caused by an accumulation of randomly occurring damage from power-line voltage spikes during electric storms, and the failure caused by normal product wear. The times are: $275,13,147,23,181,30,65,10,300,173,106$, 300, 300, 212, 300, 300, 300, 2, 261, 293, 88, 247, 28, 143, 300, 23, 300, 80, 245, and 266.

The MLEs and the maximized log-likelihood $\hat{l}_{B W}$ for the BW distribution are:

$$
\hat{a}=0.0785, \quad \hat{b}=0.0659, \quad \hat{c}=7.9355, \quad \hat{\lambda}=0.004987 \quad \text { and } \quad \hat{l}_{B W}=-169.919,
$$

whereas the MLEs and the maximized $\log$-likelihood $\tilde{l}_{W}$ for the Weibull distribution are:

$$
\tilde{c}=1.2650, \quad \tilde{\lambda}=0.005318 \quad \text { and } \quad \tilde{l}_{W}=-184.3138 .
$$

The LR statistic for testing the hypothesis $a=b=1$ (namely, Weibull versus BW distribution) is then $w=28.7896$, which indicates that the Weibull distribution should be rejected. As an alternative test, 
we can use the Wald statistic. The asymptotic covariance matrix of the MLEs for the BW distribution, which comes from the inverse of the expected information matrix, is given by

$$
\widehat{K}^{-1}=10^{-7} \times\left(\begin{array}{rrrr}
8699.35364 & 4743.69977 & -488130.870 & 87.9136383 \\
4743.69977 & 13079.4394 & -4009.69885 & -135.603333 \\
-488130.870 & -4009.69885 & 58517447.8 & -16222.8149 \\
87.9136383 & -135.603333 & -16222.8149 & 6.19530131
\end{array}\right) .
$$

The value of the Wald statistic is $W=38.4498$, again signalizing that the $\mathrm{BW}$ distribution conforms to these data. In Figure 7 we display the density of both Weibull and BW distributions fitted and the histogram of the data, where it is seen that the BW model captures the apparent bi-modality of the data.

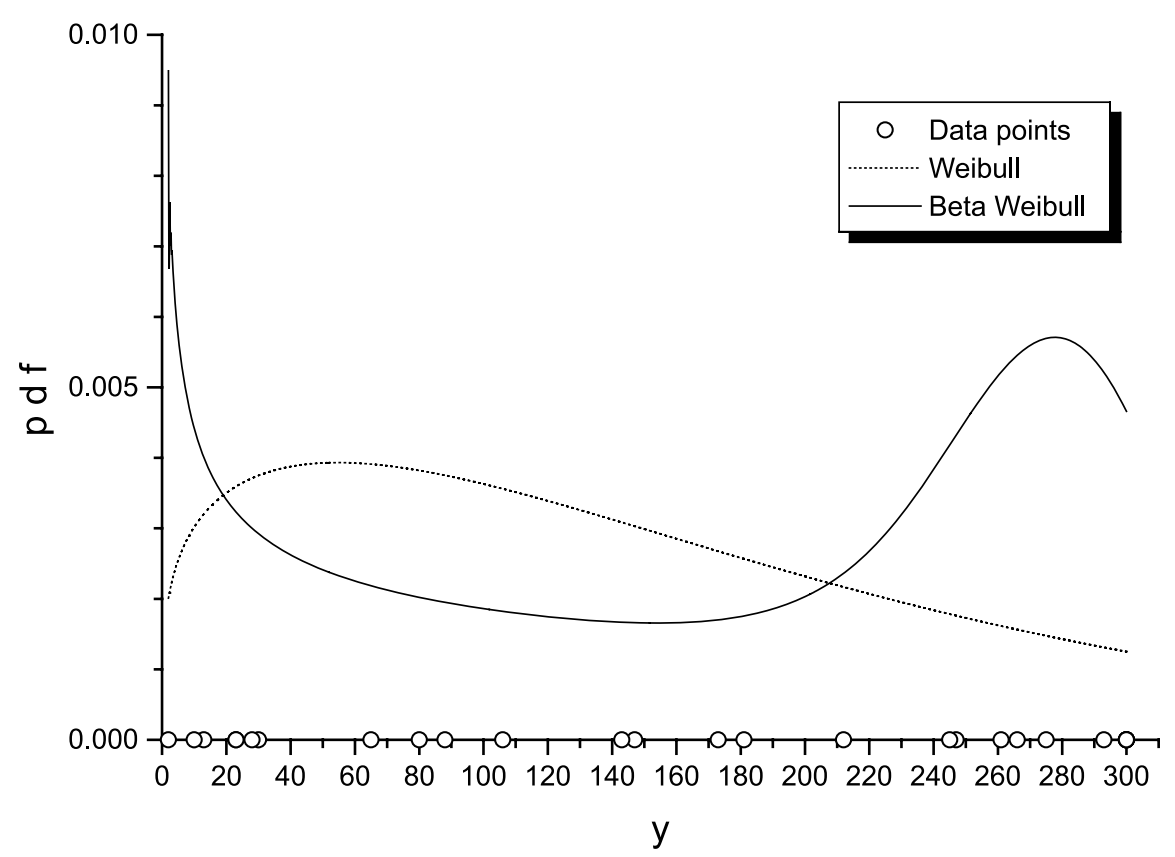

Fig. 7 - The probability density function (3) of the fitted BW and Weibull distributions.

\section{THE LOG-BETA WEIBULL DISTRIBUTION}

Let $X$ be a random variable having the BW density function (3). The random variable $Y=\log (X)$ has a log-beta Weibull (LBW) distribution whose density function, say $f(y)=f(y ; a, b, \sigma, \mu)$, which is parameterized in terms of $\sigma=c^{-1}$ and $\mu=-\log (\lambda)$, is given by

$$
f(y)=\frac{1}{\sigma B(a, b)} \exp \left\{\left(\frac{y-\mu}{\sigma}\right)-b \exp \left(\frac{y-\mu}{\sigma}\right)\right\}\left\{1-\exp \left[-\exp \left(\frac{y-\mu}{\sigma}\right)\right]\right\}^{a-1},
$$

where $-\infty<y, \mu<\infty$ and $\sigma>0$. The corresponding survival function is

$$
S(y)=1-\frac{1}{B(a, b)} \int_{0}^{1-\exp \left[-\exp \left(\frac{y-\mu}{\sigma}\right)\right]} w^{a-1}(1-w)^{b-1}=I_{1-\exp \left[-\exp \left(\frac{y-\mu}{\sigma}\right)\right]}(a, b) .
$$

We can define the standardized random variable $Z=(Y-\mu) / \sigma$ with the density function

$$
\pi(z ; a, b)=\frac{1}{\sigma B(a, b)} \exp [z-\exp (z)]\{1-\exp [-\exp (z)]\}^{a-1},
$$


for $-\infty<z<\infty$. The standard extreme-value distribution corresponds to the particular choice of $a=b=1$. A LBW regression model can be constructed from (27), and the parameters can be estimated by maximum likelihood. However, this will be conducted in a future research.

\section{CONCLUSION}

The Weibull distribution, having exponential and Rayleigh distributions as special cases, is a very popular distribution for modeling lifetime data and the phenomenon with monotone failure rates. We study the beta Weibull (BW) distribution, which represents a generalization of several distributions previously considered in the literature, such as the exponentiated Weibull (EW) distribution (Mudholkar et al. 1995, Mudholkar and Hutson 1996, Nassar and Eissa 2003, Nadarajah and Gupta 2005, Choudhury 2005) obtained for $b=1$. The Weibull distribution (with parameters $c$ and $\lambda$ ) is also another particular case for $a=1$ and $b=1$. When $a=1$, the BW distribution reduces to a Weibull distribution with parameters $\lambda b^{1 / c}$ and $c$. The beta exponential distribution is also an important special case for $c=1$.

The BW distribution provides a rather general and flexible framework for statistical analysis. It unifies several previously proposed families of distributions, therefore yielding a general overview of these families for theoretical studies, and it also provides a rather flexible mechanism for fitting a wide spectrum of real world data sets.

We derive explicit expressions for the moments of the BW distribution and an expansion for the moment generating function. We also derive the Rényi entropy. These expressions are manageable and, with the use of modern computer resources with analytic and numerical capabilities, may turn into adequate tools comprising the arsenal of applied statisticians. We discuss the estimation procedure by maximum likelihood and derive the information matrix. We demonstrate an application to real data.

We note that our analysis is related only to uncensored lifetime data and the censored case is not dealt with. We could try to extend some parts of the article to the censored situations in a future research. However, we will not obtain closed form expressions for the moments, and the integral forms will depend on numerical software. Maximum likelihood estimation and the observed information matrix using numerical methods would be a possibility.

Finally, a Bayesian analysis as developed by Cancho et al. (1999) for the EW model could possibly be extended to the more general situation of the BW distribution.

\section{ACKNOWLEDGMENTS}

We gratefully acknowledge the grants from Conselho Nacional de Desenvolvimento Científico e Tecnológico $(\mathrm{CNPq})$. The authors are also grateful to the editor and the two referees for helpful comments and suggestions.

\section{RESUMO}

A distribuição beta Weibull (BW) foi primeiramente introduzida por Famoye et al. (2005), e estudada por estes autores e Lee et al. (2007). No entanto, eles não fornecem expressões explícitas para os momentos. Neste artigo, nós obtemos expressões explícitas, em forma fechada, para os momentos desta distribuição, o que generaliza resultados 
disponíveis na literatura para alguns sub-modelos. Nós obtemos expansões para a função de distribuição acumulada e entropia de Rényi. Além disso, discutimos sobre estimação por máxima verossimilhança e fornecemos fórmulas para os elementos da matriz de informação de Fisher. Nós também mostramos a utilidade desta distribuição em um conjunto de dados reais.

Palavras-chave: distribuição Beta Weibull, matriz de informação de Fisher, máxima verossimilhança, momento, distribuição Weibull.

\section{REFERENCES}

Brown BW, Spears FM And LeVy LB. 2002. The $\log F$ : a distribution for all seasons. Computation Stat 17 : $47-58$.

CANcho VG, Bolfarine H AND AchCAR JA. 1999. A Bayesian analysis for the exponentiated-Weibull distribution. J Appl Statist 8: 227-242.

Choudhury A. 2005. A simple derivation of moments of the exponentiated Weibull distribution. Metrika 62: $17-22$.

Eugene N, Lee C and Famoye F. 2002. Beta-normal distribution and its applications. Commun Stat-Theor M 31: 497-512.

Famoye F, Lee C and Olumolade O. 2005. The Beta-Weibull Distribution. J Stat Theory Appl 4: 121-136.

Gupta AK And NAdARAJAh S. 2004. On the moments of the beta normal distribution. Commun Stat-Theor M 33: $1-13$.

JONES MC. 2004. Families of distributions arising from distributions of order statistics. Test 13: 1-43.

Lee C, Famoye F and Olumolade O. 2007. Beta-Weibull Distribution: Some Properties and Applications to Censored Data. J Mod Appl Stat Methods 6: 173-186.

Meeker WQ And Escobar LA. 1998. Statistical Methods for Reliability Data. J Wiley, New York.

Mudholkar GS, SRivastava DK and Freimer M. 1995. The exponentiated Weibull family. Technometrics 37: 436-45.

Mudholkar GS And Hutson AD. 1996. The exponentiated Weibull family: some properties and a flood data application. Commun Stat-Theor M 25: 3059-3083.

NADARAJAh S AND Gupta AK. 2005. On the moments of the exponentiated Weibull distribution. Commun Stat-Theor M 34: 253-256.

Nadarajah S And Kotz S. 2004. The beta Gumbel distribution. Math Probl Eng 10: 323-332.

NADARAJAh S And Kotz S. 2006. The beta exponential distribution. Reliab Eng Syst Safe 91: 689-697.

NASSAR MM AND EISSA FH. 2003. On the exponentiated Weibull distribution. Commun Stat-Theor M 32: $1317-1336$.

SoNG KS. 2001. Rényi information, loglikelihood and an intrinsic distribution measure. J Stat Plan Infer 93: 51-69. 


\section{APPENDIX}

In what follows, we derive the identities (16) and (17). We start from

$$
f(x)=\exp (-b x)\left(1-e^{-x}\right)^{a-1},
$$

which yields

$$
\int_{0}^{\infty} x^{d-1} f(x) d x=\int_{0}^{\infty} x^{d-1} \exp (-b x)\left(1-e^{-x}\right)^{a-1} d x,
$$

and setting $z=e^{-x}$ gives

$$
\int_{0}^{\infty} x^{d-1} f(x) d x=\int_{0}^{1}|\log z|^{d-1} z^{b-1}(1-z)^{a-1} d z .
$$

For real non-integer $a$, we have

$$
\int_{0}^{\infty} x^{d-1} f(x) d x=\Gamma(a) \sum_{j=0}^{\infty} \frac{(-1)^{j}}{\Gamma(a-j) j !} \int_{0}^{1}|\log z|^{\gamma / c-1} z^{b+j-1} d z .
$$

Also, for real $p>-1$ and real $q$, we have

$$
\int_{0}^{1} x^{p}|\log x|^{q} d x=\frac{\Gamma(1+q)}{(1+p)^{q+1}} .
$$

Hence,

$$
\int_{0}^{\infty} x^{d-1} f(x) d x=\Gamma(a) \sum_{j=0}^{\infty} \frac{(-1)^{j} \Gamma(d)}{\Gamma(a-j) j !(b+j)^{d}},
$$

and, finally, we arrive at

$$
\int_{0}^{\infty} x^{d-1} \exp (-b x)\left(1-e^{-x}\right)^{a-1} d x=\Gamma(a) \Gamma(d) \sum_{j=0}^{\infty} \frac{(-1)^{j}}{\Gamma(a-j) j !(b+j)^{d}},
$$

which represents the identity (16).

Now, let $a>0$ be an integer; then, from equation (28), we have

$$
\int_{0}^{\infty} x^{d-1} f(x) d x=\sum_{j=0}^{a-1}\left(\begin{array}{c}
a-1 \\
j
\end{array}\right)(-1)^{j} \int_{0}^{1}|\log z|^{d-1} z^{b+j-1} d z .
$$

Using (29) we obtain

$$
\int_{0}^{\infty} x^{d-1} f(x) d x=\sum_{j=0}^{a-1}\left(\begin{array}{c}
a-1 \\
j
\end{array}\right) \frac{(-1)^{j} \Gamma(d)}{(b+j)^{d}}
$$

and therefore we arrive at

$$
\int_{0}^{\infty} x^{d-1} \exp (-b x)\left(1-e^{-x}\right)^{a-1} d x=\Gamma(d) \sum_{j=0}^{a-1}\left(\begin{array}{c}
a-1 \\
j
\end{array}\right) \frac{(-1)^{j}}{(b+j)^{d}},
$$

which represents the identity (17). 